\title{
Fatores intervenientes na proteção radiológica ocupacional no controle de qualidade em câmaras gama
}

\author{
Intervening Factors in Occupational Radiological Protection in Quality Control in the \\ Gamma Cameras
}

Matheus da Silva Ventura da Conceição1, Julita Maria Freitas Coelho², Mauricio Mitsuo Monção ${ }^{3 *}$, Jaqueline de Santana Figueredo ${ }^{1}$, Claudio Henrique Santos Magalhães ${ }^{4}$, Fernando da Silva Ramos ${ }^{4}$,

Caroline Santos Silva ${ }^{5}$

\begin{abstract}
${ }^{1}$ Graduação em Tecnologia em Radiologia. IFBA; ${ }^{2}$ Docente do Departamento de Tecnologia em Saúde e Biologia. IFBA, Graduação em Odontologia. UFBA, Mestrado e Doutorado em Saúde Coletiva; ${ }^{3}$ Professor do EBTT - Instituto Federal da Bahia, Mestrado em Ciências da Saúde; ${ }^{1}$ Graduação em Tecnologia em Radiologia. IFBA. ${ }^{4}$ Tecnólogo em Radiologia, Docente de Educação Profissional em Radiologia da Escola INSSJT, Salvador/BA.; ${ }^{5}$ Gradução em Enfermagem e Pós-graduação em Saúde Pública
\end{abstract}

\begin{abstract}
Resumo
Introdução: a proteção radiológica é campo do conhecimento que permeia os ambientes radiológicos. Sua aplicação envolve benefícios aos serviços, profissionais e usuários. Objetivo: identificar fatores intervenientes, bem como, dispositivos de proteção radiológica disponíveis em um serviço de medicina nuclear (MN), envolvidos nos testes de Controle de Qualidade em Câmaras Gama (CQCG). Metodologia: realizou-se um estudo observacional, do tipo exploratório e descritivo, em um serviço de MN conveniado com o Instituto Federal da Bahia (IFBA), com ênfase na proteção radiológica em testes de CQCG. A coleta de dados foi realizada em janeiro de 2016. Foram acompanhados testes diários de inspeção visual da integridade física, radiação de fundo da sala de exames, uniformidade intrínseca e centralização da largura da janela energética para cada radionuclídeo. Para os testes semanais, acompanharam-se os de resolução espacial e linearidade. Os dados foram analisados à luz da legislação/normativas vigentes para serviços de MN no Brasil. Resultados: referente à proteção radiológica em testes de CQCG, o serviço dispunha de três aventais plumbíferos e três protetores de tireóide com equivalência de $0,5 \mathrm{~mm}$ de chumbo, além de pinças utilizadas para manipulação de fontes radioativas. Foi observada a presença de óculos de proteção, entretanto, os mesmos não eram plumbíferos. Os profissionais eram monitorados por dosímetros termoluminescentes de uso individual. Conclusão: de maneira geral, eram observados os fatores de proteção radiológica: tempo, distância e blindagem. 0 CQCG requer planejamento prévio com protocolo devidamente seguido que poderá minimizar a exposição ocupacional.

Palavras-chave: Proteção Radiológica. Medicina Nuclear. Controle de Qualidade. Câmaras Gama.
\end{abstract}

\begin{abstract}
Introduction: radiation protection is a field of knowledge that permeates radiological environments. Its application involves benefits to services, professionals and patients. Objective: To identify intervening factors, as well as the radiological protection devices available in a nuclear medicine service (MN) involved in the Quality Control tests in Gamma Camera (CQCG). Methodology: an observational, exploratory and descriptive study was carried out at the MN service in partnership with Instituto Federal da Bahia (IFBA), with emphasis on radiological protection in CQCG tests. Data collection was performed in January 2016. Daily tests were conducted to visually inspect the physical integrity of the system, to test the background radiation of the examination room, and intrinsic uniformity and centralization of the energetic window width for each radionuclide were monitored. For the weekly tests, those of spatial resolution and linearity were monitored. The data was analyzed in accordance with the legislation/regulations for MN services in Brazil. Results: with regard to the radiological protection during CQCG tests, the service had three lead aprons and three thyroid protectors with a $0.5 \mathrm{~mm}$ equivalence of lead, in addition, tweezers used to manipulate radioactive sources. The presence of protective goggles was observed, however, they did not contain lead. The professionals were monitored by thermoluminescent dosimeters for individual use. Conclusion: in general, the principles of radiation protection of time, distance and shielding were adopted. CQCG requires prior planning with a properly followed protocol that can minimize occupational exposure.

Keywords: Radiation Protection. Nuclear Medicine. Quality Control. Gamma Cameras.
\end{abstract}

\section{INTRODUÇÃO}

A proteção radiológica é um campo do conhecimento de natureza interdisciplinar. Sua aplicação pode gerar

Correspondente/Corresponding: *Mauricio Mitsuo Monção-Instituto Federal da Bahia, Tecnologia em Radiologia - End: Rua Emídio dos Santos, s/n, Barbalho, Salvador-BA. CEP 40301-015. - Tel: (71) 99176-0072.

- Email: maurimitsuo@yahoo.com.br benefícios aos serviços de radiologia em geral, para os profissionais que atuam diretamente com radiações ionizantes e usuários (MACHADO et al., 2011).

Isso é de particular importância na especialidade médica denominada Medicina Nuclear (MN), na qual a interação da energia com os tecidos biológicos produz imagens do corpo a partir do uso de radiotraçadores, geralmente na forma de radiofármacos complexos. Tais 
imagens são formadas a partir da distribuição do material radioativo empregado (THRALL; ZIESSMAN, 2006).

Essa técnica permite a aquisição de imagens funcionais (fisioanatômicas) do radioisótopo, por quantificação de funções bioquímicas e fisiológicas específicas, a partir de fótons gama gerados pelo decaimento nuclear de um volume de material radioativo. Isso se dá por dispositivos específicos, capazes de capturar a radiação emitida no local de concentração do mesmo. Dessa forma, processos patológicos podem ser detectados em fases iniciais, vez que as alterações metabólicas das doenças ocorrem antes das anomalias estruturais (HENKIN, 1996; SILVA, 2004).

No entanto, para que se obtenha uma melhor acurácia dos procedimentos em $\mathrm{MN}$, como também um controle de doses de radiação em indivíduos ocupacionalmente expostos (IOE's) e do público, se faz necessária à preservação de boas condições dos equipamentos empregados. Nesse sentido, a legislação nacional já prevê um conjunto de testes periódicos de controle de qualidade (CQ) nos equipamentos de câmaras gama (CG), além das especificações do fabricante e normas internacionais (MACHADO et al., 2011).

O CQ inclui a realização de uma série de testes de verificação das condições de operação dos equipamentos para garantir que as calibrações estejam adequadas (CHRISTIAN, 2007). Alguns destes testes, com emprego de fontes radioativas pontuais para análise de desempenho. Especificamente no caso do controle de qualidade em câmaras gama (CQCG) o tecnécio 99 metaestável é o elemento frequentemente empregado. Para a realização de tais testes, torna-se preponderante a adoção de critérios rígidos de proteção radiológica aos profissionais envolvidos frente à exposição sistemática dos mesmos a essas fontes não seladas. Sabe-se que por mínimas que sejam tais doses, seu efeito é probabilístico e aumenta com a dose de exposição (MACHADO et al., 2011).

Dessa forma, princípios básicos de proteção radiológica no manuseio, produção, posse e utilização de fontes, bem como transporte, armazenamento e deposição de materiais radioativos, foram normatizados pela Comissão Nacional de Energia Nuclear (CNEN). Por sua vez, a normativa CNEN NN 3.05 (BRASIL, 2013) estabelece os requisitos de segurança e proteção radiológica para serviços de $\mathrm{MN}$, expondo os deveres técnicos do serviço, atribuições do titular, do responsável técnico e dos indivíduos ocupacionalmente expostos (IOE's). Tal documento inclui os requisitos operacionais relativos às instalações físicas, aos instrumentos de medição da radiação, equipamentos diagnósticos e dos testes de aceitação e de controle de qualidade necessários. A mesma também agrega requisitos de proteção radiológica no recebimento das fontes radioativas, na manipulação e administração de radiofármacos, da monitoração individual e dos níveis de notificação para os IOE's, da monitoração de taxa de dose e da contaminação de superfícies, e, do preparo e administração de radiofármacos para terapia. Por fim, elenca os critérios para o gerenciamento de rejeitos radioativos e tópicos mínimos para treinamento desses profissionais (BRASIL, 2013).

Nesse sentido, se faz relevante à avaliação sistemática de materiais, equipamentos e procedimentos nos serviços de $\mathrm{MN}$ para compreender como se dá a operacionalização das estratégias de proteção radiológica. Para tanto, o objetivo desse estudo foi identificar os fatores intervenientes, bem como os dispositivos de proteção radiológica disponíveis para os técnicos e tecnólogos em radiologia em um serviço de MN, envolvidos nos testes de CQCG.

\section{METODOLOGIA}

O estudo foi desenvolvido em um serviço de $\mathrm{MN}$, de um hospital na cidade de Salvador-BA, conveniado com o Instituto Federal de Educação da Bahia. Para tanto, o projeto da presente pesquisa foi submetido à análise de viabilidade junto à coordenação do referido serviço, que autorizou sua realização, mediante assinatura de um termo de anuência à mesma.

Foi realizado um estudo observacional, do tipo exploratório e descritivo, com ênfase na proteção radiológica em testes de CQCG. A coleta de dados foi realizada durante o mês de janeiro de 2016, em horário previamente agendado antes do início dos exames com pacientes, já que os procedimentos de controle de qualidade acontecem sistematicamente nesse momento. Tais procedimentos foram acompanhados para obtenção de informações relativas à proteção radiológica, incluindo estrutura física, dispositivos de proteção e fatores intervenientes. Nessa etapa foram acompanhados testes de CQCG de rotina diária, tais como, inspeção visual da integridade física do sistema; radiação de fundo da sala de exames; uniformidade intrínseca; e, centralização da largura da janela energética para cada radionuclídeo, bem como testes de rotina semanal de resolução espacial e linearidade.

Para registros das observações, foi previamente construído um instrumento de coleta de dados do tipo roteiro com base na norma CNEN NN 3.05 de 2013. Tal instrumento incluiu identificação do serviço (natureza e tipo de atendimento); equipe técnica; distribuição das salas (quantidade, dimensão e localização); CG (disposição da sala, equipamento e mobiliário); responsável pela realização dos referidos testes; presença do livro de registros da realização dos testes; frequência da realização e tipos de testes realizados.

Seguindo as etapas programadas no instrumento de coleta de dados, inicialmente foram coletadas informações que possibilitassem a quantificação e caracterização da equipe técnica que compunha o setor. Tais dados foram adquiridos através da observação do fluxo dos profissionais e do acesso ao livro de monitoração dosimétrica, que continha a função dos profissionais do setor e sua área de atuação.

Em seguida, dados referentes à distribuição espacial dos ambientes foram observados e devidamente registrados. A quantidade das salas foi averiguada, bem 
como sua localização. As dimensões foram obtidas com o auxílio de uma trena milimetrada, com quatro metros de comprimento. Por último, os dispositivos de proteção radiológica utilizados nos testes de CQCG foram observados considerando as suas finalidades específicas.

Para análise dos dados obtidos, tomou-se como referência a Norma CNEN NN 3.05 do ano de 2013, referente à temática deste trabalho, a RDC n. 38 (BRASIL, 2008) e RDC n. 50 (BRASIL, 2002). Além desses, utilizaram-se outros documentos como referências bibliográficas disponíveis na literatura especializada.

\section{RESULTADOS}

A equipe técnica do serviço era composta por três médicos nucleares, dois profissionais físicos, dois tecnólogos em radiologia, quatro técnicos em radiologia, um enfermeiro, cinco técnicos em enfermagem e dois técnicos em higienização. Os testes de CQCG incluídos na rotina diária foram realizados pelos profissionais técnicos ou tecnólogos em radiologia durante a semana de coleta dos dados. Já os testes da rotina semanal foram realizados uma única vez por um profissional em física.

Quanto à estrutura física o serviço apresentava dois consultórios médico/enfermagem, um laboratório de radiofarmácia, sala de infusão de radiofármacos, sala de pacientes injetados e duas salas de exames onde estão instaladas e operando as CG. Todas as salas estavam identificadas com "acesso restrito" e o símbolo internacional das radiações ionizantes, exceto os dois consultórios.

A sala de exame, identificada como Sala 01, dispunha da CG linha NM 600, modelo Discovery 630 do fabricante General Electric. Já a sala de exame, identificada como Sala 02, a CG era o modelo FORTE, SPECT 3/8, fabricado pela PHILIPS. Quanto às dimensões das salas, foram observadas as medidas de $4 \mathrm{~m} \times 5,77 \mathrm{~m}$ para a sala $1 \mathrm{e} 3,60 \mathrm{~m}$ $\times 5,77 \mathrm{~m}$ para a sala 2 . Observaram-se também algumas diferenças na estrutura física e no comando de operação das CG que são características próprias de cada modelo de equipamento.

Para a realização dos testes de uniformidade intrínseca, centralização da largura da janela energética, resolução espacial e linearidade foi separada uma fonte pontual de tecnécio 99 metaestável de $600 \mu \mathrm{Ci}$ devidamente medida em uma câmara de ionização do tipo poço modelo CRC-25 do fabricante Carpintec. Após o devido preparo, esta fonte foi conduzida até a sala da GC e fixada na parede a uma distância de 2 metros do equipamento e a 1 metro de altura, tomando como base o solo.

Ao final da realização de cada um dos testes de CQGC, os respectivos resultados eram registrados pelo profissional em livros próprios do setor.

No tocante aos dispositivos de proteção radiológica individual, foi averiguado que os profissionais envolvidos utilizavam os dosímetros de tórax e os dosímetros de extremidade. Também, havia um total de cinco dosímetros para monitoração de área no setor. Além disso, os dispositivos empregados pelo serviço eram três aventais plumbíferos e três protetores de tireóide, ambos com equivalência de $0,5 \mathrm{~mm}$ de chumbo, uma capela blindada para preparo da fonte radioativa, três pinças metálicas para manuseio dos recipientes contendo a referida fonte, e um recipiente de chumbo para transporte das mesmas.

Figura 1 - Posicionamento da fonte pontual de tecnécio 99 metaestável

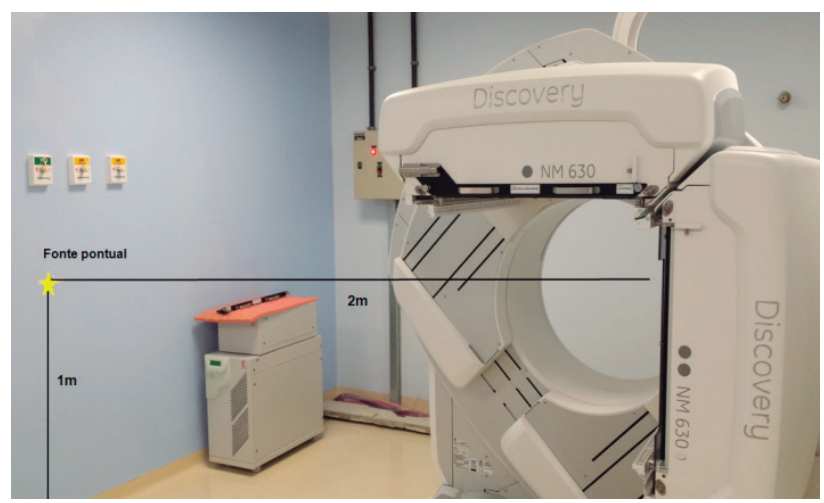

Fonte: Pesquisa (2016).

Por fim, foram identificados como fatores intervenientes à proteção radiológica de IOE's, o não uso de óculos plumbíferos para proteção do cristalino, a variação de tempo na preparação e/ou manipulação da fonte radioativa pontual entre os profissionais envolvidos nessa atividade e a permanência desta fonte dentro da sala após o término dos testes de CQCG.

\section{DISCUSSÃO}

O serviço de MN estudado, de forma geral, seguia os pressupostos normativos relativos à proteção radiológica profissional em testes de CQCG. Tais testes podem ser úteis na antecipação de necessidades de reparação dos equipamentos do serviço, favorecendo a manutenção da rotina de trabalho preestabelecida, sem interrupções do fluxo normal para possíveis reparos. Além disso, ao minimizar a possibilidade de repetição de exames, favorece indiretamente a redução de doses em IOE e do público.

Em relação aos ambientes, a RDC n. 50 (BRASIL, 2002) estabelece que o setor de MN deva ser composto por: laboratório de manipulação e estoque de fontes em uso; sala de decaimento; box para coleta de material; laboratório de radioimunoensaio; sala de administração de radiofármacos; sala ou box de pacientes injetados; sala de exames; sala de laudos e arquivos; quarto para internação com banheiro exclusivo para pacientes tratados com lodo-131. Esses pressupostos foram reafirmados por Záu (2008), que identificou as necessidades do espaço físico para o serviço de MN. Destaca-se aqui que o serviço dispunha de todos esses ambientes, organizados segundo a RDC acima citada, o que possibilita a fluidez das práticas desenvolvidas no referido setor. 
Além desses, a norma da CNEN NN 3.05 (BRASIL, 2013) indica ainda que os seguintes ambientes devam fazer parte do setor de MN: sala de espera para pacientes, sanitário exclusivo para pacientes, local para armazenamento dos rejeitos radioativos, laboratório de manipulação e armazenamento de fontes em uso, sala para administração de radiofármacos, sala de exames e quarto com sanitário privativo para internação de paciente com dose terapêutica. Foi observado no serviço investigado que todos os ambientes estavam em conformidade com as especificações das legislações de referência.

Em referência às salas de $C G$, o serviço dispunha de salas com dimensões apropriadas e de acordo com o estabelecido pela RDC n. 50 (BRASIL, 2002) que preconiza uma área média de $25 \mathrm{~m}^{2}$. A grandeza distância tem um forte efeito na redução da dose, pois a taxa de dose é inversamente proporcional ao quadrado da distância. Por isso, também é importante o uso de pinças para o manuseio de frascos. Além disso, sempre manipular o material radioativo atrás da blindagem e manter os frascos contendo materiais radioativos sempre blindados. Devem-se usar transportadores blindados para fontes sempre que necessário (MACHADO et al., 2011).

Quanto aos testes semanais de inspeção visual da integridade física do sistema, radiação de fundo da sala de exames, uniformidade intrínseca, centralização da largura da janela energética para cada radionuclídeo, foram executados de acordo com as indicações da norma CNEN NN 3.05 (BRASIL, 2013). No entanto, para o teste realizado semanalmente (linearidade e resolução espacial), é sugerido por esta norma sua execução mensal. Embora as normas vigentes indiquem a periodicidade mínima para a realização dos testes, deve-se considerar que o aumento nas repetições destes acarretará também o aumento da exposição dos IOE's.

O controle de constantes físicas da sala de exames, embora não seja um teste realizado na CG, merece receber também uma atenção especial. Isso se deve ao fato de que o controle tanto da temperatura da sala de exames quanto da umidade relativa do ar pode ser determinante na condição de funcionamento das CG, tendo em vista a característica higroscópica dos cristais cintiladores, peculiaridade essa apontada por Thrall e Ziessman (2006).

A CNEN NN 3.05 (BRASIL, 2013) preconiza ainda que os testes de CQ com periodicidade inferior à semestral devem ser realizados por profissionais da equipe de tecnologistas devidamente treinados e indicados pelo titular do serviço. No serviço estudado, profissionais técnicos e tecnólogos em radiologia realizaram os referidos testes, enquanto aqueles de periodicidade superior ficavam a cargo do especialista em física médica responsável pelo setor. Destaca-se que o teste de resolução espacial e linearidade foram realizados apenas uma vez na semana pelo profissional especialista. Especialmente em relação à inspeção visual, segundo Souza (2014), esta deve incluir a observação de danos, desgastes, limpeza, lubrificação e funcionamento de componentes mecânicos que atuam no movimento de todos os componentes do equipamento, a exemplo da mesa de exames, detectores, rotação do gantry, sistema de troca de colimadores quando adequado, dentre outros. Dessa forma podem ser verificados possíveis danos em componentes, acessórios, cabos externos, funcionamento de botões de emergência e monitores de posicionamento. Em síntese, as condições ambientais (temperatura e umidade) do local onde o equipamento esteja instalado, juntamente com eventuais variações na rede elétrica, podem comprometer o funcionamento e desempenho do equipamento (SOUZA, 2014). Essa inspeção visual era rotineiramente realizada no serviço deste estudo.

Vale ainda salientar que para os testes acima citados é necessário que todo o procedimento seja planejado antes da execução e que todo cuidado deve ser tomado para evitar a contaminação com material radioativo em quaisquer dos componentes da CG. Para tanto, o uso de luvas de procedimentos utilizadas na separação da fonte pontual de tecnécio 99 metaestável no laboratório deve ser descartada e outra luva deve ser utilizada para manuseio de quaisquer componentes/objetos na sala da GC, o que também afirma Machado et al. (2011). Nesse sentido, ressalta-se que uma equipe capacitada, periodicamente submetida a treinamentos e atualização, tem papel fundamental na obtenção de resultados positivos na proteção radiológica.

Dentre os equipamentos de proteção individual e de proteção coletiva devem estar disponíveis luvas de procedimentos, jalecos de manga longa, óculos plumbíferos, transportadores blindados para seringas e frascos, pinças com dimensões adequadas ao tipo de manipulação, blindagem para manipulação, transporte e armazenamento de fontes radioativas e rejeitos (BRASIL, 2013). Em relação aos aventais plumbíferos, a maioria atualmente comercializada possui espessura de $0,25 \mathrm{~mm}$ e $0,5 \mathrm{~mm}$, oferecendo proteção satisfatória para isótopos radioativos de baixas energias como o tecnécio 99 metaestável utilizado nos testes de CQCG (MACHADO et al., 2011; SOARES; PEREIRA; FLÔR, 2011).

No processo de trabalho dos testes, ocorre a exposição à radiação ionizante dos profissionais. É considerado como risco físico e se apresenta de forma invisível, existindo sempre a probabilidade de ocorrência de dano à saúde dos IOE's (MACHADO; FLÔR; GELBCKE, 2009).

Ressalta-se que outros equipamentos de proteção radiológica eram disponibilizados no serviço investigado, tais como blindagens para deslocamento de seringas e janelas de transição, embora não se apliquem diretamente ao processo de CQCG. No entanto, foi observado que durante o período de coleta não houve a utilização dos óculos com proteção plumbífera. Logo, uma exposição direta do cristalino pode ter ocorrido, o que certamente contribuiu para o aumento de valores de dosimetria dos IOE's.

Essa preocupação já motivou a publicação pela Comissão Internacional de Proteção Radiológica (ICRP) 
de redução de valores do limiar de dose absorvida para efeitos tardios nos cristalinos (ICRP, 2007). Nesse quesito, a norma CNEN NN 3.01 (BRASIL, 2014) normatiza a redução do limite de dose equivalente do cristalino para 20 $\mathrm{mSv}$ por ano durante 5 anos, não excedendo $50 \mathrm{mSv}$ em nenhum ano. No entanto é importante ressaltar que o tempo de observação do serviço em análise foi de apenas cinco dias o que impossibilita uma avaliação mais apurada do não uso dos óculos plumbíferos.

Ainda em relação à proteção radiológica profissional, observou-se que a monitoração dosimétrica dos profissionais foi devidamente realizada, pois todos envolvidos nos testes de CQCG portavam dosímetros termoluminescentes de tórax e extremidades. Isso foi um achado muito relevante na avaliação geral do rigor do controle de qualidade implantado no serviço investigado, pois, o principal meio de avaliação da eficiência de um programa de controle de dose estabelecido e dos procedimentos adotados no serviço é a dosimetria individual (TAUHATA et al., 2013).

Em relação a tais dispositivos, ressalta-se que os dosímetros são de uso individual e apenas devem ser utilizados durante a jornada de trabalho. Quando verificado um nível de dose efetiva acima do limiar especificado pela CNEN, deve-se investigar o motivo que a ocasionou. Mesmo havendo margem de tolerância, deve-se buscar sempre a menor dose possível (SILVA et al., 2014).

Quanto aos fatores intervenientes na proteção radiológica profissional durante os procedimentos de CQCG foi verificado que os intervalos de tempo dispendidos no manuseio e posicionamento da fonte pontual de tecnécio 99 metaestável poderiam ser mais uniformes. Detectou-se variabilidade na duração de tempo para preparação e/ou manipulação da fonte pontual entre os profissionais envolvidos. Isso suscita a possibilidade de padronização de tempo considerando os intervalos menores. Segundo Machado et al., (2011), o tempo de exposição pode ser diminuído executando as atividades necessárias o mais rápido possível junto às fontes radioativas. Haramita (2011) reforça a necessidade de treinamentos específicos visando melhorias das condições de proteção radiológica.

Ainda, em relação a tal fonte, verificou-se que depois de preparada, era conduzida do laboratório de manipulação até a sala da CG e posicionada pelo profissional de radiologia. Após a realização dos testes de CQCG, a fonte pontual permaneceu na sala de exames contribuindo também para uma exposição adicional do profissional e dos pacientes no momento do exame, bem como, no aumento da radiação de fundo. Sabe-se que após a utilização da fonte, esta deve ser armazenada ou descartada em local apropriado. A literatura aponta que assim se pode evitar exposição desnecessária. Isso foi corroborado pelo entendimento de Tauhata et al. (2013), que afirmam não existir um limiar real de dose para os efeitos estocásticos. Dessa forma, também se evita produção de eventuais artefatos de imagem devido à radiação emitida por essa fonte dentro da sala de exames (MACHADO et al., 2011).

\section{CONCLUSÃO}

De forma geral, a proteção radiológica em MN garante o uso seguro das fontes de radiação não seladas, considerando o progressivo aumento dos procedimentos médicos que fazem uso de radiação ionizante. Sabe-se que a MN, por utilizar pequenas quantidades de material radioativo, é classificada como atividade de baixo risco. No entanto, são necessários cuidados especiais na manipulação e no descarte criterioso dos materiais radioativos utilizados, inclusive, aqueles utilizados durante a realização de testes de CQCG.

O serviço estudado, de uma forma geral, pode ser considerado seguro para IOE's e usuários. No entanto, sugere-se revisão/adoção de um planejamento prévio, particularmente com vistas à redução da exposição ocupacional. Por isso, é imprescindível um protocolo de trabalho para os testes de controle de qualidade, que devidamente seguido, poderá otimizar os procedimentos realizados. Dessa forma, favorecer uma maior segurança e efetividade concorrendo para uma práxis mais segura.

\section{AGRADECIMENTOS}

Os autores do trabalho agradecem o apoio financeiro da PRPGI/IFBA e ao Departamento de Tecnologia e Saúde e Biologia - DTSBio.

\section{REFERÊNCIAS}

BRASIL. Agência Nacional de Vigilância Sanitária (ANVISA). RDC 38 : Instalação e funcionamento de serviços de medicina nuclear "in vivo". Diário Oficial [da] União, Brasília, 2008.

. Agência Nacional de Vigilância Sanitária (ANVISA). Ministério da Saúde. RDC 50: Técnico para planejamento, programação, elaboração e avaliação de projetos físicos de estabelecimentos assistenciais de saúde. Diário Oficial [da] União, Brasília, 2002.

Norma Nuclear CNEN NN 3.05. Requisitos de segurança e proteção radiológica para serviços de medicina nuclear. Diário Oficial [da] União, Brasília, 2013.

. Norma Nuclear CNEN NN 3.01. Diretrizes básicas de proteção radiológica. Diário Oficial [da] União, Brasília, 2014.

. Instituto de pesquisa energéticas e nucleares (IPEN). Radioproteção e dosimetria: fundamentos. Rio de Janeiro: Instituto de Radioproteção e Dosimetria, Comissão Nacional de Energia Nuclear, 2013.

CHRISTIAN, P. E. PET instrumentation. In: NUCLEAR medicine and PET/ $\mathrm{CT}$ technology and techniques. 6 ed. St. Louis, Missouri: Mosby Elsevier, 2007. p. 333-336.

HARAMITA, T. Aplicação dos princípios de radioproteção em medicina nuclear. 2011. Dissertação (Mestrado) - Instituto de Biociências, UNESP, Botutcatu, 2011.

HENKIN, R. E. Nuclear medicine. St. Louis, Missouri: Mosby, 1996.

INTERNATIONAL COMMISSION ON RADIOLOGICAL PROTECTION (ICRP). The 2007 recommendations of the International Commission on Radiological Protection. 2007. 332 p.

MACHADO, M. A. D. et al. Revisão: radioproteção aplicada à medicina nuclear. Rev. Bras. Fis. Med., São Paulo, v. 4, n. 3, p. 47-52, 2011.

MACHADO, R. R.; FLÔR, R. C.; GELBCKE, F. L. Educação Permanente: 
Uma estratégia para dar visibilidade aos riscos físicos e biológicos. Rev. Saúde Públ. Santa Catarina, Florianópolis, v. 2, n. 1, p. 30-40, 2009.

SILVA, F. L. et al. Avaliação da dose efetiva acumulada em três clínicas de medicina nuclear. In: CONGRESSO BRASILEIRO DE ENGENHARIA BIOMÉDICA-CBEB, 24., 2014, Uberlândia. Anais...Uberlândia: SBEB , 2014.

SILVA, M. T. S. Correção de Atenuação em Imagens Simuladas de Perfusão Cardíaca em SPECT. 2004. 75 f. Trabalho de Conclusão (Graduação em Física) - Faculdade de Física, PUCRS, Porto Alegre, 2004.

SOARES, F. A. P; PEREIRA, A. L.; FLÔR, R. C. Utilização de vestimentas de proteção radiológica para redução de dose absorvida: uma revisão integrativa da literatura. Rev. Bras. Radiol., São Paulo, v. 44, n. 2, p. 97-103, 2011.

SOUZA, J. S. Avaliação do programa de controle de qualidade e segu- rança dos equipamentos PET instalados em regiões do Brasil. 2014. Dissertação (Mestrado em Engenharia Biomédica) - Programa de Pós Graduação em Engenharia Biomédica, Faculdade Gama, Universidade de Brasília, Brasília, DF, 2014. 110 p.

TAUHATA, L. et al. Radioproteção e dosimetria: fundamentos. Instituto de radioproteção e dosimetria, comissão nacional de energia nuclear. Rio de Janeiro, 2013.

THRALL, J. H.; ZIESSMAN, H. A. Medicina nuclear. 2 ed. Rio de Janeiro: Guanabara Koogan, 2006.

ZÁU, M. A. Necessidades do espaço físico em unidade de medicina nuclear. 2008. 54f. Trabalho Final (Especialização) - Programa de Pós-Graduação em Arquitetura, Faculdade de Arquitetura, Universidade Federal da Bahia, 2008.

Submetido em: $13 / 01 / 2017$

Aceito em: 30/03/2017 